\title{
Center for
}

Energy and

Environmental

Economic

Studies

\section{Lucas Bretschger}

\section{Alexandra Vinogradova}

Growth and Mitigation Policies with Uncertain Climate Damage

Working paper CE3S-02/14

St. Petersburg

2014 


\section{ББК 65.012.2}

B85

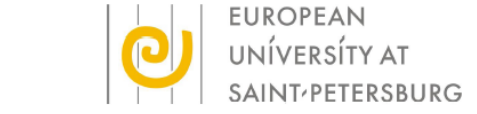

European University at St. Petersburg

Department of Economics

\section{CORE}

Université Catholique de Louvain

Center for Operations Research and Econometrics

Center for Energy and Environmental Economic Studies

\section{Bretschger L., Vinogradova A.}

B85 Growth and Mitigation Policies with Uncertain Climate Damage / Lucas Bretschger, Alexandra Vinogradova: CEEES paper CE3S-02/14; Center for Energy and Environmental Economic Studies. St. Petersburg: EUSP, 2014. — 41 p.

We analyze an endogenously growing economy in which production generates greenhouse gas emissions leading to global temperature increase. Global warming causes stochastic climate shocks, modeled by the Poisson process, which destroy part of the economy's capital stock. Part of the output may be devoted to emissions abatement and thus damages from climate shocks may be reduced. We solve the model in closed form and show that the optimal path is characterized by a constant growth rate of consumption and capital stock until a shock arrives, triggering a downward jump in both variables. The magnitude of the jump depends on the Poisson arrival rate, abatement efficiency, damage intensity, and the elasticity of intertemporal consumption substitution. Optimum mitigation policy consists of spending a constant share of output on abatement, which is an increasing function of the Poisson arrival rate, the economy's productivity, polluting intensity of output, and the intensity of environmental damage. Optimum growth and abatement react sharply to changes in the arrival rate and the damage intensity, suggesting more stringent climate policies for a realistic world with uncertainty compared to the certainty-equivalent case. We extend the baseline model by adding climate-induced fluctuations around the growth trend and stock pollution effects, showing the robustness of our results.

Lucas Bretschger, Center of Economic Research, CER-ETH, Zurich, Switzerland. Tel: +41-44632-21-92. E-mail: lbretschger@ethz.ch.

Alexandra Vinogradova, Center of Economic Research, CER-ETH, Zurich, Switzerland. Tel: +41-44-632-21-92. E-mail: avinogradova@ethz.ch.

Издание осуществлено за счет средств проекта создания специализации по природным ресурсам и экономике энергетики «ЭксонМобил» 


\title{
Growth and Mitigation Policies with Uncertain Climate Damage
}

\author{
Lucas Bretschger and Alexandra Vinogradova*
}

May 28, 2014

\begin{abstract}
We analyze an endogenously growing economy in which production generates greenhouse gas emissions leading to global temperature increase. Global warming causes stochastic climate shocks, modeled by the Poisson process, which destroy part of the economy's capital stock. Part of the output may be devoted to emissions abatement and thus damages from climate shocks may be reduced. We solve the model in closed form and show that the optimal path is characterized by a constant growth rate of consumption and capital stock until a shock arrives, triggering a downward jump in both variables. The magnitude of the jump depends on the Poisson arrival rate, abatement efficiency, damage intensity, and the elasticity of intertemporal consumption substitution. Optimum mitigation policy consists of spending a constant share of output on abatement, which is an increasing function of the Poisson arrival rate, the economy's productivity, polluting intensity of output, and the intensity of environmental damage. Optimum growth and abatement react sharply to changes in the arrival rate and the damage intensity, suggesting more stringent climate policies for a realistic world with uncertainty compared to the certainty-equivalent case. We extend the baseline model by adding climate-induced fluctuations around the growth trend and stock pollution effects, showing the robustness of our results.
\end{abstract}

JEL Classification: O10, Q52, Q54

Key Words: Climate policy, uncertainty, natural disasters, endogenous growth.

${ }^{*}$ Both Center of Economic Research, CER-ETH, Zurich, Switzerland. Tel: +41-44-632-21-92, email: lbretschger@ethz.ch, avinogradova@ethz.ch 


\section{Introduction}

\subsection{Economics and the Climate}

Economic models are usually successful in reducing the pervasive complexity of modern economies to a low number of well-specified analytical relationships. This allows to study the basic mechanics of an economic problem and the associated policy options in a concise and clear manner. When it comes to the analysis of climate change, several factors seem to impede the procedure severely. Both the economic and the ecological part of the climate problem pose considerable modeling challenges, involving long time horizons and various sources of uncertainty. As a consequence, the vast majority of current economic climate frameworks consists of relatively complex numerical simulation models. These have provided many useful insights about costs and benefits of a climate policy but also produced diverging results. Hence, to gain further insights about the central mechanisms at work, a climate economic model providing closed-form solutions for future growth and optimum climate policy appears to be very desirable.

Understanding the economic effects of climate change is not only desirable but necessary and urgent. The global temperature escalation is predicted to intensify the severity of natural disasters, increasingly harming development in different parts of the world. Ferocious tropical hurricanes, massive floods, droughts and landslides cause severe destruction of infrastructure, loss of physical and human capital, and undoubtedly a setback in terms of economic growth. According to National Oceanic and Atmospheric Administration (2013), the cost of extreme weather events has risen from about $\$ 20 \mathrm{bn}$ in 1980 s to almost $\$ 90$ bn in 2010 . The recent Typhoon Haiyan in the Philippines was the strongest recorded storm to make landfall ever (see The Economist (2013)). The surge swept away entire cities, at least $11 \mathrm{~m}$ Filipinos have been affected, some killed, many displaced or left homeless. Although climate physicists are not unanimous on whether the frequency of natural disasters will increase in the future or not, the majority agrees that the intensity will get worse as the planet warms (see IPCC (2007)). It is well understood that economic activities cause carbon and other greenhouse gas emissions which deteriorate 
the natural environment and lead to cliamte change. However, occurrence of climate shocks is not easily predictable and is typically viewed as a random event.

Given the uncertain feature of natural disasters caused by climate change, how should an economy appropriately balance its production, consumption, investment, and reduction of emissions? How to determine the optimal growth rate and the optimal abatement in the uncertain environment? And finally, how do these key variables respond to changes in the underlying economic fundamentals? In the present paper we address these questions within a model of a growing economy which features uncertainty about the arrival of natural disasters. We assume that the occurrence of a natural disaster (also referred to as an "event") follows a random process, and when an event happens to occur, part of the economy's capital stock is destroyed. It follows that the capital accumulation process is both endogenous and stochastic. We also assume that the magnitude of the damage to the capital stock is a function of polluting emissions, which appears to be a crucial feature of climate change. However, in our model the world does not end after an environmental disaster, as it is often assumed in the literature on catastrophic events (see Section 1.3). We consider development with recurring shocks over time, which reflects a likely pattern of climate-induced events in the future. Reduction in emissions, and therefore the extent of climate damages, can be achieved by appropriately balancing two types of activities: capital accumulation and abatement.

\subsection{Basic results}

To the best of our knowledge the paper is the first to provide a clear-cut closed form solution for the optimal abatement expenditure and the growth rate of the economy subject to uncertain climate shocks with endogenous damages. We show that the optimal policy consists of devoting a constant share of output to emissions abatement. We also show that a more frequent occurrence of natural disasters (i.e., higher arrival rate) and a higher intensity of events have a negative impact on the optimal growth and call for a more vigorous abatement policies. These findings support the general intuition and the validity of our approach. We show that the optimal consumption and the capital stock 
grow at the same rate until an event arrives, causing a downward jump in both variables. The size of the jump is endogenously determined and depends on the arrival rate, abatement efficiency, damage intensity and the intertemporal substitution elasticity. As an illustration of an optimal path, we show in Figure 1a the log-consumption rate as a function of time. The solid line represents the stochastic path, which exhibits a growth rate $g$ in case of no climate event. At times $t_{1}$ and $t_{2}$, negative environmental shocks are assumed to occur, causing an immediate downward jump, followed by a subsequent period of growth with the previous rate. We provide a complete characterization of the growth rate and the size of the jump. The dashed line shows the time profile of consumption under expected growth rate. As can be seen from the figure (and will be derived in detail below), that consumption growth under uncertainty is unambiguously higher than under the expected equivalent and reflects a specific kind of the broadly known precautionary effect. The gap between the effective and expected growth relates not only to the optimal capital investment but also to the optimal climate mitigation activities, which are the focus of the current climate policy discussions. Figure 1a concisely illustrates one of our main arguments: There is a fundamental difference between the dashed and the solid curves in that the former smoothes out the jumps and discontinuities of the latter, thereby ignoring the crucial effects of uncertainty. Given that uncertain shocks constitute a central part of the economic analysis of climate change, they need to be taken into account within an appropriate framework of analysis.

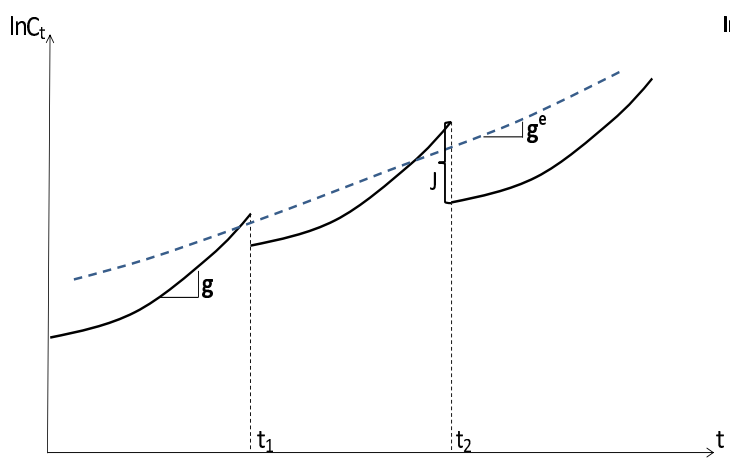

(a) Stochastic vs expected path.

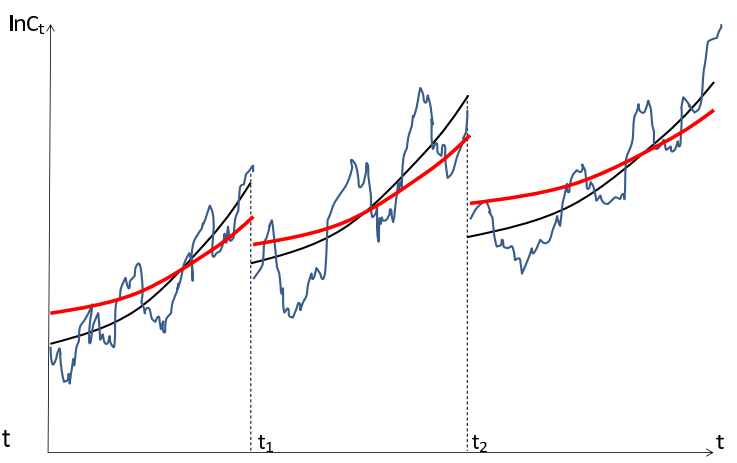

(b) Extended models.

Figure 1: ... 
In the final part of the paper, we provide two extensions of the baseline setup by introducing (i) climate change induced fluctuations around the growth trend, modeled by a Brownian motion, in addition to the Poisson-driven jumps and (ii) the link between climate damage size and the entire history of pollution. Figure $1 \mathrm{~b}$ provides an illustration of the optimal log-consumption path under these two alternative scenarios, where the former is shown by the blue line and the latter by the red line. We show that the results and intuitions of the baseline model continue to hold in these richer frameworks. In the former case, the optimal growth rate of the economy may either exceed or fall short of the growth rate in the baseline model. The share of output devoted to abatement is, however, unambiguously larger. In the second extension, the growth rate is smaller, the jumps in the consumption rate and the capital stock are also less pronounced, while the abatement share is larger than in the baseline.

\subsection{Contribution to literature}

Our paper relates to several contributions in the field of climate economics. Recently, Ikefuji and Hori (2012) examined the optimal growth rate and the carbon tax in an economy where private capital is subject to stochastic depreciation due to climate change. They assumed, however, that these stochastic shocks are idiosyncratic and reflect a large number of independent small climate events. With this setup, it becomes possible to work with the expected values, while in our approach we make a sharp distinction between the expected and stochastic outcomes. As emphasized by Pizer (1999), ignoring uncertainty may lead to significant policy errors. Using an integrated assessment framework, he finds that uncertainty causes a preference for taxes over rate controls, resulting in welfare gains, and also encourages a more stringent climate policy, the result which is also confirmed in our setting. ${ }^{1}$

\footnotetext{
${ }^{1}$ Some of the integrated assessment models of climate change have recently also included different forms of uncertainty in their numerical simulation approaches, see, e.g., Pizer (1999), Ikefuji et al. (2010), Jensen and Traeger (2011), Lontzek and Narita (2011), Cai et al. (2013), and Lemoine and Traeger (2013). Contrary to these papers, we provide closed form model solution showing the exact impact of all the parameters. In addition, we do not limit ourselves to so-called "tipping points" of climate which exclude a return to regular growth but allow instead for multiple catastrophes, which we think to be a more realistic setup.
} 
Golosov et al. (2014) derived a simple formula for the optimal carbon tax showing that it is proportional to GDP and depends on just a small number of parameters. The convenient expression for the tax arises due to two simplifying assumptions: first, the future path of climate damages is constant over time; and second, the optimal saving rate is constant. Although their model is more detailed in several aspects than ours, i.e., energy production and carbon cycle, their results are similar in spirit to ours, in the sense that our climate policy instrument can also be conveniently expressed as a fraction of output which depends on the economy's fundamental characteristics. The novelty and strength of our setup is to fully characterize the solution under uncertainty about big natural disasters, which lies at the heart of the climate problem. Our framework explicitly introduces uncertainty about future climate events via the Poisson process. Moreover, the damages are not only time-varying but fully endogenous. We do not need to assume constancy of the saving rate but, instead, we derive the optimal saving rate of the economy and show how it is affected by the climate change and the associated uncertainty.

The present paper builds on two further strands of literature. The first consists of theoretical papers integrating pollution and climate change into macroeconomic models of growing economies. ${ }^{2}$ These contributions focus on growth issues under pollution constraints as in our approach. All of them, however, remain within the deterministic framework, excluding the central topic of uncertainty by assumption.

The second strand of the literature relates to optimal consumption-investment choices and environmental policy under uncertainty. Soretz (2007) analyzes efficient pollution taxation within an endogenous growth model where environmental quality has a stochastic impact on factor productivity, which is driven by a Wiener process. There is a growing literature on uncertain catastrophic events causing an irreversible damage. Among the

\footnotetext{
${ }^{2}$ See Bovenberg and Smulders (1995), Withagen (1995), Michel and Rotillon (1995), Grimaud et al. (2007), van der Ploeg and Withagen (2010), Bretschger and Valente (2011), Rezai et al. (2012), Bretschger and Suphaphiphat (2013), and the survey of Brock and Taylor (2005). In a recent paper, Bommier et al. (2013) derive solutions for a model of economic growth, stock pollution, and endogenous risk of a catastrophic collapse, showing that multiplicative preferences recommend a tighter policy response to climate change than traditional additive preferences.
} 
early contributions, Clarke and Reed (1994) show that, when uncertainty is exogenous and the event causes an irreversible catastrophic damage, optimal emissions and total pollution stock are higher than in the case of certainty. Tsur and Zemel (1996) characterize the optimal trajectory of the emissions when the pollution level, at which an undesirable irreversible event occurs, is uncertain but endogenous, depending on the entire history of pollution. They show that, contrary to Clarke and Reed (1994), the economy chooses a more conservationist path and its emissions are lower than in the certainty case. Tsur and Zemel (1998) extend the analysis to (uncertain endogenous) reversible events. Zeeuw and Zemel (2012) provide a dynamic characterization of optimal emission policy when the time of the regime switch from low to high damage is uncertain. One of their key findings is that, due to precautionary reasons, emissions may be lower than in the case where the system is already in the high-damage regime. They also discuss the trade-offs between mitigation and adaptation policies. The present paper parallels the work of Tsur and Zemel (1998) in the sense that we consider uncertain natural disasters which are, in general, multiple and reversible. We add to this literature by explicitly characterizing the optimal abatement policy aimed at curbing current emissions and thus reducing the damages caused by natural disasters. Unlike Tsur and Zemel (1998), who study the optimal steady state policy and transitional dynamics of an economy which is not engaged in any investment activity, we consider a growing economy which does indeed invest in capital accumulation and spends on abatement activities. We provide clear-cut analytical solutions for the optimal climate policy and consumption growth rate in the presence of event uncertainty with event-associated damage being a function of investment decisions.

The remainder of the paper is organized as follows. Section 2 develops the baseline framework. In Section 3, we present the main results with respect to the optimal growth rate and abatement. Section 4 extends the baseline model by introducing stock pollution and climate-induced trend fluctuations. Finally, Section 5 concludes. 


\section{The Framework}

\subsection{General model}

We consider a global economy which produces a composite consumption good under constant returns to scale using as input broadly defined capital, denoted by $K_{t}$. The production process is polluting: every period $t$ a flow of greenhouse gas emissions, denoted by $E_{t}$, is released into the atmosphere. Emissions cause deterioration of the natural environment and global temperature increase, leading to a random occurrence of natural disasters. We assume that the arrival of a natural disaster (we shall also refer to it as an "event") follows the Poisson process ${ }^{3}$ with the mean arrival rate $\lambda$. When an event occurs, an endogenously-determined amount $\gamma_{t}$ of the existing capital stock is destroyed. In fact, recent floods like the one in Pakistan in 2010 or in the Philippines in 2013 had a profound effect on the economies' infrastructure and the capital stock (both physical and human). According to the predictions of climate sciences, the magnitude of the damage is very likely to increase in the future due to climate change and hence we model it as a positive function of the economy's emissions, i.e., $\frac{\partial \gamma_{t}}{\partial E_{t}}>0$.

The output, denoted by $Y_{t}\left(K_{t}\right)$, can be either spent on consumption, $C_{t}$, or invested. There are two types of non-consumption spending: investment to augment the capital stock and financing of emissions abatement. Specifically, we assume that a share $\theta_{t}$ of output is spent on the latter, so that abatement expenditure is given by $I_{t}=\theta_{t} Y_{t}$. The remaining share $\left(1-\theta_{t}\right) Y_{t}$ is split between consumption and capital accumulation. Total abatement, $Z\left(I_{t}\right)$, is a positive function of the abatement expenditure, $Z^{\prime}\left(I_{t}\right)>0$. The total per period emissions are then given by emissions stemming from the economic activity minus abatement. We assume that one unit of output causes $\phi \in(0,1)$ units of pollution, so that total emissions are given by $E_{t}=\phi Y_{t}-Z\left(I_{t}\right)$.

The economy's objective is to maximize the expected value of utility over an infinite planning horizon with respect to consumption, $C_{t}$, and the share of output devoted to

\footnotetext{
${ }^{3}$ Modeling occurrence of natural disasters by a Poisson process is quite standard, especially in the risk and insurance literature. See, e.g., Batabyal and Beladi (2001) and Baryshnikov et al. (2001).
} 
abatement, $\theta_{t}$, subject to the stochastic capital accumulation process. Specifically, the planner's programme is

$$
\begin{gathered}
\max _{C_{t}, \theta_{t}} \mathbb{E}_{0}\left\{\int_{0}^{\infty} U\left(C_{t}\right) e^{-\rho t} d t\right\} \\
\qquad \begin{array}{l}
d K_{t}=\left[\left(1-\theta_{t}\right) Y_{t}\left(K_{t}\right)-C_{t}\right] d t-\gamma\left(E_{t}, K_{t}\right) d q_{t}, \\
E_{t}=\phi Y_{t}\left(K_{t}\right)-Z\left(I_{t}\right) \\
I_{t}=\theta_{t} Y_{t}\left(K_{t}\right),
\end{array}
\end{gathered}
$$

where $\mathbb{E}_{0}$ is the expectations operator, $d q_{t}$ is an increment of the Poisson process with a constant arrival rate $\lambda$ and $\rho$ is the rate of time preference. We also require that the capital stock, consumption and emissions are non-negative and $\theta_{t} \in[0,1)$.

\subsection{Specific Assumptions}

We now introduce some specific functional forms and explain their motivation and consequences in the model.

Assumption 1: $Y_{t}=A K_{t}$

Following the main part of economic literature we use the assumption of constant returns to scale in aggregate production. Since capital is the only input in the model, output is produced with an $A K$ technology, where $A$ is the constant factor productivity parameter and $K_{t}$ is interpreted as a broad measure of capital in the economy. We thus employ a broadly accepted model of endogenous growth literature.

Assumption 2: $Z\left(I_{t}\right)=\sigma I_{t}$

Total abatement is directly proportional to the financial means allocated to emissions control, with the proportionality parameter $\sigma>0$ representing the efficiency of abatement technology. In accordance with the linearity assumption for output it is sensible to equally assume constant returns for abatement activities.

Assumption 3: $\gamma\left(E_{t}, K_{t}\right)=\gamma_{e} E_{t}+\bar{\gamma} K_{t}$

We assume that the damage to the capital stock, $\gamma\left(E_{t}, K_{t}\right)$, consists of two terms: one which is directly proportional to emissions (the endogenous effect) and the other, 
reflecting capital destruction during a natural disaster at the rate $\bar{\gamma} \in(0,1)$ even in the absence of polluting emissions (the exposure effect). We shall refer to the parameter $\gamma_{e}$ as the damage intensity or the damage sensitivity to the economy's emissions.

Assumption 4: $\phi<\sigma<\left(\lambda \gamma_{e}\right)^{-1}$

The assumption requires the abatement productivity, $\sigma$, to be sufficiently high (to exceed polluting intensity $\phi$ ) but also to be bounded from above, given the severity of natural disasters (i.e., the Poisson arrival rate $\lambda$ and the damage intensity of climate shocks $\gamma_{e}$ ). The second restriction prevents overly optimistic technology perspectives biasing the results in a too favorable direction.

Assumption 5: $U(C)=\frac{C^{1-\varepsilon}}{1-\varepsilon}$

The utility function takes a standard CRRA form, where $1 / \varepsilon$ is the intertemporal substitution elasticity. We shall reveal the important impact of this substitution elasticity in a climate context, which is absent when log-utility is assumed.

\subsection{Solving the model}

Denoting by $V(K)$ the value function associated with the optimization problem above, the Hamilton-Jacobi-Bellman (HJB) equation may be written as

$$
\rho V(K)=\max \left\{U(C)+V^{\prime}(K)[(1-\theta) Y-C]+\lambda[\tilde{V}(\tilde{K})-V(K)]\right\}
$$

where $\tilde{V}$ is the value function after the occurrence of the event which depends on the new capital stock $\tilde{K}=K-\gamma(E, K)$. Time subscripts are omitted when there is no ambiguity. The first-order conditions are

$$
\begin{aligned}
C: & U^{\prime}(C)-V^{\prime}(K)=0 \\
\theta: & -V^{\prime}(K) Y+\lambda \tilde{V}^{\prime}(\tilde{K}) \gamma_{e} \sigma Y=0, \\
K \quad: \quad & \rho V^{\prime}(K)=V^{\prime \prime}(K)[(1-\theta) Y-C]+V^{\prime}(K) A(1-\theta)+ \\
& \lambda\left(\tilde{V}^{\prime}(\tilde{K})\left[1-\gamma_{e}(\phi-\sigma \theta) A-\bar{\gamma}\right]-V^{\prime}(K)\right) .
\end{aligned}
$$


The optimality conditions are complemented by the transversality condition for $K$, the non-negativity constraints on $C, K, E$, and the requirement $\theta \in[0,1)$. Eqs. (6) - (8) allow to obtain an explicit solution for the law of motion of the consumption rate (the derivations are relegated to the Appendix A.1)

$$
\frac{d C}{C}=\frac{1}{\varepsilon}\left\{A\left(1-\frac{\phi}{\sigma}\right)+\frac{1-\bar{\gamma}}{\sigma \gamma_{e}}-\rho-\lambda\right\} d t+\left(\frac{\tilde{C}}{C}-1\right) d q
$$

where the new consumption rate at the time of the jump, $\tilde{C}$, is a constant fraction of the pre-jump rate:

$$
\tilde{C}=\left(\lambda \sigma \gamma_{e}\right)^{\frac{1}{\varepsilon}} C
$$

with $0<\left(\lambda \sigma \gamma_{e}\right)^{\frac{1}{\varepsilon}}<1$ (see Assumption 4). It follows that the last term on the RHS is negative; it represents the downward jump in consumption every time a natural disaster occurs and destroys $\gamma_{t}$ units of the capital stock.

The first term on the RHS of (9) represents what we label the "trend" consumption growth. Specifically, while the event has not arrived, consumption grows at the constant rate, defined as

$$
g \equiv \frac{1}{\varepsilon}\left\{A\left(1-\frac{\phi}{\sigma}\right)+\frac{1-\bar{\gamma}}{\sigma \gamma_{e}}-\rho-\lambda\right\}
$$

The expression reveals that the consumption rate is increasing over time if the effective discount rate, which includes not only the pure rate of time preference $\rho$ but also the disaster arrival rate $\lambda$, is not too high, formally $g>0 \Leftrightarrow A\left(1-\frac{\phi}{\sigma}\right)+\frac{1-\bar{\gamma}}{\sigma \gamma_{e}}>\rho+\lambda$. As soon as an event occurs, consumption jumps down to the new level, $\widetilde{C}$, and then continues to grow at the rate $g$ until the next event.

It can be shown that the value function of the problem, satisfying the HJB equation and certain limiting conditions (see, e.g., Sennewald and Wälde 2006), is of the form

$$
V(K)=\frac{\psi^{-\varepsilon} K^{1-\varepsilon}}{1-\varepsilon}
$$


where

$$
\psi \equiv \frac{1}{\varepsilon}\left\{\rho-(1-\varepsilon)\left[\frac{1-\bar{\gamma}-\left(\lambda \sigma \gamma_{e}\right)^{\frac{1}{\varepsilon}}}{\sigma \gamma_{e}}+A \frac{\sigma-\phi}{\sigma}\right]+\lambda\left[1-\left(\lambda \sigma \gamma_{e}\right)^{\frac{1-\varepsilon}{\varepsilon}}\right]\right\}
$$

Proposition 1: The solution of the maximization problem given by (1) - (4) is characterized by the following:

(i) optimal consumption is a constant fraction of the capital stock;

(ii) optimal abatement expenditure is a constant fraction of output;

(iii) consumption, capital stock, output, and abatement grow at the same constant rate, given by (11).

Proof: The result in (i) follows immediately from (6) and (12), so that:

$$
C^{*}=\psi K
$$

Statement (ii) follows from (7) and (12); by combining the two expressions we find that optimal abatement is given by:

$$
\theta^{*}=\frac{\phi}{\sigma}-\frac{1-\bar{\gamma}-\left(\lambda \sigma \gamma_{e}\right)^{\frac{1}{\varepsilon}}}{A \sigma \gamma_{e}}
$$

The non-negativity constraint on $E$ requires that $\theta^{*} \leqslant \frac{\phi}{\sigma}$ (see (4) and (3)). At the same time, $\theta^{*}$ must be non-negative, so that both conditions lead to the inequality $0 \leqslant \frac{\phi}{\sigma}-\frac{1-\bar{\gamma}-\left(\lambda \sigma \gamma_{e}\right)^{\frac{1}{\varepsilon}}}{A \sigma \gamma_{e}} \leqslant \frac{\phi}{\sigma}$. After some rearrangements, we obtain

$$
1-\bar{\gamma}-A \phi \gamma_{e} \leqslant\left(\lambda \sigma \gamma_{e}\right)^{\frac{1}{\varepsilon}} \leqslant 1-\bar{\gamma}
$$

which is the necessary restriction on the parameters of the model to ensure the existence of an interior solution.

To prove (iii), note that the law of motion for the capital stock can be found explicitly by substituting the optimal controls (13) and (14) in (2) and solving the resulting 
stochastic differential equation

$$
d K_{t}=\left[\left(1-\theta^{*}\right) A-\psi\right] K_{t} d t-\left[\gamma_{e}\left(\phi-\sigma \theta^{*}\right) A+\bar{\gamma}\right] K_{t} d q_{t} .
$$

The solution is given by

$$
K_{t}=K_{0} e^{\left[\left(1-\theta^{*}\right) A-\psi\right] t+\ln \left[1-\bar{\gamma}-\gamma_{e}\left(\phi-\sigma \theta^{*}\right) A\right] q_{t}} .
$$

We can verify that the term in the exponent involving the logarithm is well-defined since the argument of the logarithm is unambiguously positive, because it is equal to (using (14))

$$
1-\bar{\gamma}-\gamma_{e}\left(\phi-\sigma \theta^{*}\right) A=\left(\lambda \sigma \gamma_{e}\right)^{\frac{1}{\varepsilon}}>0
$$

Moreover, substituting the solution for $\theta^{*}$ in $\left[\left(1-\theta^{*}\right) A-\psi\right]$, we obtain

$$
K_{t}=K_{0} e^{g t+\frac{1}{\varepsilon} \ln \left(\lambda \sigma \gamma_{e}\right) q_{t}}
$$

so that consumption and capital grow at the same rate as long as an event has not arrived, in line with (13). Abatement expenditure evolves over time according to

$$
I_{t}=\left[\frac{A \phi}{\sigma}-\frac{1-\bar{\gamma}-\left(\lambda \sigma \gamma_{e}\right)^{\frac{1}{\varepsilon}}}{\sigma \gamma_{e}}\right] K_{0} e^{g t+\frac{1}{\varepsilon} \ln \left(\lambda \sigma \gamma_{e}\right) q_{t}}
$$

showing that it grows at the trend rate $g$.

In order to better understand the role of uncertainty, we also compute the expected consumption growth rate, defined as

$$
g^{e} \equiv \frac{d \mathbb{E}_{t} C_{t}}{C_{t}}=\frac{1}{\varepsilon}\left\{A\left(1-\frac{\phi}{\sigma}\right)-\frac{\bar{\gamma}}{\sigma \gamma_{e}}-\rho+\lambda\left[\frac{1}{\lambda \sigma \gamma_{e}}+\varepsilon\left(\lambda \sigma \gamma_{e}\right)^{1 / \varepsilon}-1-\varepsilon\right]\right\} .
$$

It can be easily verified that the expected consumption growth rate $g^{e}$ is smaller than the trend growth rate: $g^{e}<g$. The log-consumption paths corresponding to the growth rates $g$ and $g^{e}$ are illustrated in Figure 1a by the solid and the dashed lines, respectively. 
We assume that climate shocks arrive at times $t_{1}$ and $t_{2}$ causing instantaneous downward jumps followed by a next period of growth. The dashed line - the hypothetical time profile of consumption corresponding to the growth rate $g^{e}$ - is flatter than the stochastic path as it smoothes out the jumps and discontinuities of the latter, ignoring the precautionary motive for saving. Therefore, drawing policy recommendations from the analysis of expected paths instead of the true stochastic paths will lead to an underestimation of both savings and emissions-control efforts. The argument is parallel to Pizer's (1999) point that we have already cited in the Introductory section. Pizer, however, relied on numerical methods to support his statement, while we provide a rigorous analytical support. Given that stochastic shocks constitute a central part of the economic analysis of climate change, they need to be taken into account within an appropriate modeling framework. The next sections propose a detailed characterization of the solution and an analysis of how the optimal growth rate and the abatement share respond to changes in the key variables of the model.

\section{Characterizing the Solution}

\subsection{Consumption Growth}

We have established in Eq. (11) that the trend growth rate of consumption is given by $g$, which we rewrite as

$$
g=\frac{1}{\varepsilon}\left\{A\left(1-\frac{\phi}{\sigma}\right)-\rho+\lambda\left(\frac{1}{\lambda \sigma \gamma_{e}}-1-\frac{\bar{\gamma}}{\lambda \sigma \gamma_{e}}\right)\right\}
$$

The expression has a familiar Keynes-Ramsey form albeit with some modifications. The standard Keynes-Ramsey growth rate equals the difference between the real interest rate (usually the marginal product of capital) and the rate of pure time preference, adjusted by the elasticity of intertemporal consumption substitution. First, note that in Eq. (18) the economy's implicit real interest rate, given by the first term inside the parentheses, 
is not equal to just the marginal productivity of capital but is reduced by the emission intensity of output, adjusted by the abatement efficiency, i.e, the term $\phi / \sigma$. It follows that in our framework, pollution has an unambiguously negative growth effect. It may be dampened by either increasing the abatement efficiency, $\sigma$, or decreasing the polluting intensity, $\phi$. Second, there is, of course, the effect of uncertainty, represented by the last term, which includes the exposure and the jump components. The exposure component, $\frac{\bar{\gamma}}{\lambda \sigma \gamma_{e}}$, is present due to our assumption that natural catastrophes may occur even in the absence of any polluting activity. In that case, arrival of a climate shock causes $\bar{\gamma}$ percent damage to the existing capital stock. This effect contributes to a growth slow-down. On the other hand, the jump component $\frac{1}{\lambda \sigma \gamma_{e}}$ translates into a faster trend consumption growth as compared to the standard Keynes-Ramsey growth. Since $\frac{1}{\lambda \sigma \gamma_{e}}$ is the ratio of marginal utilities of post- to pre-jump consumption, it is larger than unity (see also Eqs. (10) and (15)) and thus the term $\frac{1}{\lambda \sigma \gamma_{e}}-1$ is positive. The faster growth is possible only when the economy has a relatively low consumption rate at the beginning of the planning horizon, which implies an initial phase of precautionary saving, including expenditures on abatement. The result is analogous to what has been found in the literature on precautionary savings under uncertainty. ${ }^{4}$ In the current setting we allow for two types of non-consumption expenditures: investment in capital and financing of abatement activities, with the optimal split between the two being determined endogenously.

The responses of the economy's optimal growth rate to changes in the fundamental parameters of the model are summarized in Proposition 2. It is important to distinguish between the effect of the expected frequency of natural disasters and the effect of the overall uncertainty. The former takes into account only the arrival rate $\lambda$. The latter includes both the arrival rate and the damage caused by the occurrence of an event, as reflected in the last term in Eq. (18).

Proposition 2: Assuming Poisson uncertainty about the arrival of climate shocks, we establish the following effects:

\footnotetext{
${ }^{4}$ See, e.g., Wälde (1999), Toche (2001), Steger (2005).
} 
(i) higher expected frequency of events, higher polluting intensity of production, and higher damage intensity are associated with a lower optimal growth rate,

(ii) higher factor productivity contributes to a faster growth,

(iii) higher abatement efficiency has an ambiguous effect on growth.

Proof: Follows from comparative statics (Eq. (18)):

$$
\begin{aligned}
& \frac{\partial g}{\partial \lambda}=-\frac{1}{\varepsilon}<0, \quad \frac{\partial g}{\partial \phi}=-\frac{A}{\varepsilon \sigma}<0, \quad \frac{\partial g}{\partial \gamma_{e}}=-\frac{1-\bar{\gamma}}{\varepsilon \sigma \gamma_{e}^{2}}<0, \\
& \frac{\partial g}{\partial A}=\frac{1}{\varepsilon}\left(1-\frac{\phi}{\sigma}\right)>0, \quad \frac{\partial g}{\partial \sigma}=\frac{1}{\varepsilon \sigma^{2}}\left(A \phi-\frac{1-\bar{\gamma}}{\gamma_{e}}\right) \gtrless 0 .
\end{aligned}
$$

The effect of the arrival rate $\lambda$ on the optimal growth is directly proportional to the negative of the elasticity of intertemporal consumption substitution. Although there is not a general consensus on the magnitude of this elasticity, the empirically plausible range of values lies between 1 and 3 . This suggests that if the frequency of natural disasters were to rise in the future due to accentuated climate change, the economy may experience an important growth slowdown. The results on polluting intensity $\phi$ and damage intensity $\gamma_{e}$ are intuitive and have already been discussed. The fact that a higher abatement efficiency has an ambiguous bearing on economic growth is due to two effects - the emissions-reduction effect and the jump-smoothing effect - which work in the opposite directions. On the one hand, an improvement in efficiency of abatement reduces total emissions and thus enhances the growth rate through the first term in Eq. (18). On the other hand, it increases the post-event consumption rate, shrinking the pre- to post-event consumption gap (see Eq. (10)) and thus contributes to a growth slowdown through the last term in Eq. (18). If the overall productivity or polluting intensity or climate damage sensitivity are large, then the effect of $\sigma$ on the trend growth rate is positive. This suggests that economies with a relatively high polluting intensity of production (higher $\phi$ ) and highly exposed to climate shocks (higher $\left.\gamma_{e}\right)$, such as developing economies, may enjoy substantial gains in terms of their growth rates by adopting (more) efficient abatement technologies. At the same time, economies 
with a relatively higher total factor productivity and a larger capital stock (such as advanced economies) may also experience an increase in the growth rate by improving their abatement technologies.

\section{$3.2 \quad$ Abatement}

How much emissions to abate and how much of the current resources to devote to emissions control are key policy questions. We have shown in the previous section that in our model it is optimal to allocate a specific constant fraction of output to abatement activities. The solution for the abatement share $\theta^{*}$ is reproduced from Eq. (14) for convenience

$$
\theta^{*}=\frac{\phi}{\sigma}-\frac{1-\bar{\gamma}}{A \sigma \gamma_{e}}+\frac{\left(\lambda \sigma \gamma_{e}\right)^{\frac{1}{\varepsilon}}}{A \sigma \gamma_{e}}
$$

It is worth noting that optimal abatement share is independent of the discount rate, which is a broadly debated parameter in climate economics. Climate policy recommendations usually differ substantially depending on the assumed discount rate, ${ }^{5}$ while our policy result is robust in this respect. ${ }^{6}$ We now examine and discuss how the optimal abatement share depends on the fundamental parameters of the model.

Proposition 3: Assuming Poisson uncertainty driving the arrival of climate events, we establish the following:

(i) abatement share is an increasing function of the event arrival rate, total factor productivity, polluting intensity of output, and damage intensity,

(ii) abatement share may be either decreasing or increasing in abatement efficiency, depending on the parameter constellation.

\footnotetext{
${ }^{5}$ For instance, Nordhaus (2000) uses $1.5 \%$ per year discount rate to calculate the carbon tax of $\$ 30$ per ton coal. Stern (2007) uses a much lower discount rate of $0.1 \%$ and estimates the tax to be $\$ 250$.

${ }^{6}$ Note that this result will change once we introduce pollution as a stock in the next Section.
} 
Proof: The results can be obtained from the following comparative statics, using Eq. (19)

$$
\begin{aligned}
& \frac{\partial \theta^{*}}{\partial \lambda}=\frac{\left(\lambda \sigma \gamma_{e}\right)^{\frac{1}{\varepsilon}-1}}{A \varepsilon}>0, \quad \frac{\partial \theta^{*}}{\partial A}=\frac{1-\bar{\gamma}-\left(\lambda \sigma \gamma_{e}\right)^{\frac{1}{\varepsilon}}}{A^{2} \sigma \gamma_{e}}>0, \quad \frac{\partial \theta^{*}}{\partial \phi}=\frac{1}{\sigma}>0 \\
& \frac{\partial \theta^{*}}{\partial \gamma_{e}}=\frac{1+\frac{1-\varepsilon}{\varepsilon}\left(\lambda \sigma \gamma_{e}\right)^{\frac{1}{\varepsilon}}-\bar{\gamma}}{A \sigma \gamma_{e}^{2}}>0, \quad \frac{\partial \theta^{*}}{\partial \sigma}=\frac{1-A \phi \gamma_{e}+\frac{1-\varepsilon}{\varepsilon}\left(\lambda \sigma \gamma_{e}\right)^{\frac{1}{\varepsilon}}-\bar{\gamma}}{A \sigma^{2} \gamma_{e}} \gtrless 0 .
\end{aligned}
$$

The statements in (i) are intuitive. An increasing event arrival rate $\lambda$ requires more abatement in order to better protect the economy against climate damages. If policy makers happened to misperceive the true arrival rate $\lambda$, the abatement policy would be sub-optimal. Specifically, if the predicted $\lambda$ is lower than the true $\lambda$, there is too little abatement. This might happen if climate change induces a regime switch from low to high event frequency but general expectations lag because they are based on past experience.

The total factor productivity $(A)$ fosters pollution by raising output and thus acts in the same direction as the pollution parameters, such as polluting intensity of output $(\phi)$ and damage intensity $\left(\gamma_{e}\right)$.

The statement in (ii) warrants some further comments. The reason for the ambiguous sign in $\partial \theta^{*} / \partial \sigma$ is that there are three effects which operate in different directions. They can be analyzed by examining the expression in (19). First, there is a direct effect of $\sigma$ on the optimal abatement share, operating through the first term on the RHS of (19): Better abatement technology requires a smaller expenditure on emissions reduction, all else equal. Second, a better abatement efficiency has a positive effect on the economy's growth rate (provided $A \phi \gamma_{e}>1-\bar{\gamma}$ ), which in turn calls for a larger abatement expenditure to compensate for an increase in polluting activities. Finally, abatement efficiency also affects the size of the downward jump in the consumption rate and in the capital stock when an adverse event occurs (the last term in (19)). The direction of this latter effect, however, depends on the intertemporal substitution elasticity, $1 / \varepsilon$. When it is relatively high (resp., low), i.e., above (resp., below) unity, the effect of $\sigma$ on the downward jump is positive (resp., negative). Overall, the first (direct) effect contributes to a decrease in abatement share; the second (growth) effect contributes to an increase in abatement 
share; while the third (jump) effect can be either positive or negative, depending on the intertemporal substitution elasticity.

Lemma 1: If the intertemporal substitution elasticity is above (below) unity,

(i) the optimal abatement share is convex (concave) in the arrival rate;

(ii) the response of the abatement share to a change in the arrival rate is more (less) pronounced when abatement technology is more (less) efficient and when climate-damage intensity is larger (smaller).

Proof: Follows directly from

$$
\begin{aligned}
\frac{\partial^{2} \theta^{*}}{\partial \lambda^{2}} & =\left(\frac{1}{\varepsilon}-1\right) \frac{\lambda^{\frac{1}{\varepsilon}-2}\left(\sigma \gamma_{e}\right)^{\frac{1}{\varepsilon}-1}}{A \varepsilon} \gtrless 0 \Leftrightarrow \frac{1}{\varepsilon} \gtrless 1, \\
\frac{\partial^{2} \theta^{*}}{\partial \lambda \partial \sigma} & =\left(\frac{1}{\varepsilon}-1\right) \frac{\sigma^{\frac{1}{\varepsilon}-2}\left(\gamma_{e} \lambda\right)^{\frac{1}{\varepsilon}-1}}{A \varepsilon} \gtrless 0 \Leftrightarrow \frac{1}{\varepsilon} \gtrless 1, \\
\frac{\partial^{2} \theta^{*}}{\partial \lambda \partial \gamma_{e}} & =\left(\frac{1}{\varepsilon}-1\right) \frac{\gamma_{e}^{\frac{1}{\varepsilon}-2}(\sigma \lambda)^{\frac{1}{\varepsilon}-1}}{A \varepsilon} \gtrless 0 \Leftrightarrow \frac{1}{\varepsilon} \gtrless 1 .
\end{aligned}
$$

The Lemma implies that, when the frequency of natural disasters is already relatively high, a further increase in the frequency should be associated with a more (less) than proportional increase in abatement if the intertemporal substitution elasticity is greater (smaller) than unity.

Lemma 2: If the intertemporal substitution elasticity is

(i) below 3, then the optimal abatement share is concave in the damage intensity;

(ii) below 2, then the response of the abatement share to a change in the damage intensity is less pronounced when abatement technology is more efficient. (These conditions are sufficient but not necessary.)

Proof: Follows directly from

$$
\begin{aligned}
\frac{\partial^{2} \theta^{*}}{\partial \gamma_{e}^{2}} & =\frac{\left(\lambda \sigma \gamma_{e}\right)^{\frac{1}{\varepsilon}}(1-3 \varepsilon)+2 \varepsilon^{2}\left[\left(\sigma \lambda \gamma_{e}\right)^{\frac{1}{\varepsilon}}-(1-\bar{\gamma})\right]}{A \sigma \gamma_{e}^{3}} \gtrless 0, \\
\frac{\partial^{2} \theta^{*}}{\partial \gamma_{e} \partial \sigma} & =\left(\frac{1-\varepsilon}{\varepsilon}\right)^{2} \frac{\left(\lambda \sigma \gamma_{e}\right)^{\frac{1}{\varepsilon}}}{A \sigma^{2} \gamma_{e}^{2}}-\frac{1-\bar{\gamma}}{A \sigma^{2} \gamma_{e}^{2}} \gtrless 0 .
\end{aligned}
$$


These results formally support the argument that it is optimal to increase abatement activities when the magnitude of climate change related damages or the expected frequency of natural disasters increase. Our model predicts that the optimal increase in abatement share should be more (less) than proportional to an increase in the frequency of events if the intertemporal substitution elasticity is relatively high (low). The intuition here is straightforward. A higher elasticity of intertemporal consumption substitution implies that the economy is easily willing to forgo current consumption in exchange for more consumption in the future and thus increase current abatement. In the limiting case $\varepsilon=1$ (logarithmic utility), $\theta^{*}$ is linear in $\lambda$ and monotone-increasing and concave in the damage intensity, $\frac{\partial^{2} \theta^{*}}{\partial \gamma_{e}^{2}}=-\frac{2(1-\bar{\gamma})}{A \sigma \gamma_{e}^{3}}<0$. It can be either monotone-decreasing and convex or monotone-increasing and concave in abatement efficiency, depending on $A \phi \gamma_{e} \gtrless 1-\bar{\gamma}$.

\subsection{Implications for Propensity to Save}

The fraction of output devoted to abatement represents a part of the economy's gross propensity to save, which we denote by $s$. The effect of climate change on $s$ is of great interest from the macroeconomic perspective. Our gross savings consist of two types of expenditures: investment in capital accumulation and financing of abatement activities, with the optimal split between the two endogenously determined. Using Eq. (13), we may express $s$ as

$$
s=1-\frac{\psi}{A}=\frac{1}{A \varepsilon}\left\{A-\rho+(1-\varepsilon) \frac{1-\bar{\gamma}-\left(\lambda \sigma \gamma_{e}\right)^{\frac{1}{\varepsilon}}-A \phi \gamma_{e}}{\sigma \gamma_{e}}-\lambda\left[1-\left(\lambda \sigma \gamma_{e}\right)^{\frac{1-\varepsilon}{\varepsilon}}\right]\right\} .
$$

First note that $s$ depends only on the parameters of the model and does not depend on time. The optimality of a time-invariant propensity to save is a useful result which justifies the constant propensity to save assumption in Golosov et al. (2014), allowing the authors to derive their simple formula for the carbon tax. Second, with a log-utility assumption the expression simplifies to $1-\frac{\rho}{A}$ and thus excludes the climate-related 
parameters altogether. This simplified preference structure implies that climate change would only cause a reallocation between investment and abatement, but not between consumption and gross savings. When $\varepsilon$ is different from unity, the effects of the key climate parameters on $s$ are as follows:

$$
\begin{aligned}
& \frac{\partial s}{\partial \lambda}=\frac{1}{A \varepsilon}\left\{\left(\lambda \sigma \gamma_{e}\right)^{\frac{1}{\varepsilon}-1}-1\right\} \gtrless 0 \Leftrightarrow \frac{1}{\varepsilon} \lessgtr 1, \\
& \frac{\partial s}{\partial \phi}=-\frac{1-\varepsilon}{\varepsilon \sigma} \gtrless 0 \Leftrightarrow \frac{1}{\varepsilon} \lessgtr 1, \\
& \frac{\partial s}{\partial \gamma_{e}}=\frac{(1-\varepsilon)}{A \varepsilon} \frac{\left(\lambda \sigma \gamma_{e}\right)^{\frac{1}{\varepsilon}}}{\sigma \gamma_{e}^{2}} \gtrless 0 \Leftrightarrow \frac{1}{\varepsilon} \gtrless 1, \\
& \frac{\partial s}{\partial \sigma}=\frac{(1-\varepsilon)}{A \varepsilon} \frac{\left(\lambda \sigma \gamma_{e}\right)^{\frac{1}{\varepsilon}}+A \phi \gamma_{e}}{\sigma^{2} \gamma_{e}} \gtrless 0 \Leftrightarrow \frac{1}{\varepsilon} \gtrless 1 .
\end{aligned}
$$

The value of the intertemporal substitution elasticity appears to be crucial for resolving the ambiguity in the climate effects. For instance, an increase in $\lambda$ causes an unambiguous increase in $\theta$ but may lead to a decline in $s$ if $1 / \varepsilon$ is relatively high (above unity) and to a decline in $s$ if $1 / \varepsilon$ is relatively low (smaller than unity). It follows that, when the elasticity is relatively high, the optimal response of the economy to an increase in disaster frequency is to increase both its abatement expenditure and the current consumption at the expense of capital accumulation. By contrast, when the elasticity is relatively low, an increase in the abatement share is accompanied by a reduction in both the current consumption and capital accumulation $\left(\frac{\partial s}{\partial \lambda}<\frac{\partial \theta}{\partial \lambda}\right.$, see the exact expression for $\frac{\partial \theta}{\partial \lambda}$ in the proof of Proposition 2). Moreover, when $\varepsilon$ approaches unity, the derived impact of all the parameters - and most importantly those related to climate change, $\lambda$ and $\gamma_{e}$ - are at the lower bound of the empirically plausible impact range.

\subsection{Quantitative Implications}

In this subsection we illustrate the quantitative implications of our model by comparing the implied optimal abatement share with recent findings in the literature. For instance, Golosov et al. (2014) calculate the optimal carbon tax of $\$ 56.9$ per ton carbon assuming a discount rate of $1.5 \%$ (as in Nordhaus 2000) and the world output of $\$ 70$ trillion in 
2010. Given the total world emissions of about 9.7 bn tons of carbon in that year, the total tax proceeds would amount to $0.8 \%$ of world output. Assuming a ten times lower discount rate (as, for instance, suggested by Stern 2007) would imply an approximately ten times higher carbon tax and the total proceeds of about $8 \%$ of world output - a quite significant number.

The abatement share in our model is independent of the discount rate and thus more robust to subjective perceptions of this parameter. However, it depends on a set of parameters, some of which may be more easily calibrated than others, although we remain cautious about choosing values pertaining to the world economy as a whole. We shall set our reference unit of time to one year and use averages over the past decade to calibrate specific parameters. We set $\bar{\gamma}$ to 0.001 and $A$ to 0.04 . For calculating the growth rate we assume the rate of time preference of $1.5 \%$ per year, as in Golosov et al. (2014) and Nordhaus (2000). The former article also assumes a unitary elasticity of substitution, $\varepsilon=1$, which we adopt here as well. The statistics for large-scale natural disasters over the last two decades suggest that the arrival rate $\lambda \approx 0.0993$ - event arrives approximately once in ten years. We also know that damages from big natural catastrophes constitute approximately $0.9 \%$ of output. ${ }^{7}$ This proxies for the consumption drop equal to $1-\left(\lambda \sigma \gamma_{e}\right)^{1 / \varepsilon}$ in our model. We can then calibrate $\sigma \gamma_{e}$ as $1-\left(\lambda \sigma \gamma_{e}\right)^{1 / \varepsilon}=$ $0.009=>\sigma \gamma_{e}=(0.991)^{\varepsilon} / \lambda$. With $\lambda=0.0993$ and $\varepsilon=1$, we obtain $\sigma \gamma_{e}=9.9798$. The last parameter to calibrate is the polluting intensity of output $\phi$. Polluting intensities vary considerably not only across countries but also across industries and even firms in the same industry within a given country. As a starting point, we set $\phi=0.05$ and test robustness of our results with respect to variations in this parameter.

Under the benchmark calibration described above, we obtain the optimal abatement share $\theta$ of 0.8968 percent and the trend growth rate of consumption of 3.4552 percent. Our value of $\theta$ of $\approx 0.9 \%$ is slightly higher, although comparable, to the $0.8 \%$ implied

\footnotetext{
${ }^{7}$ For example, the Indian Ocean Tsunami in 2004 caused at least $\$ 10$ bn worth of damage and affected mainly six countries: Indonesia, India, Maldives, Sri Lanka, Somalia, and Thailand. The damage amounts to $0.86 \%$ of the sum of GDPs in 2004 of the affected countries (Somalia not included due to lacking GDP data in WDI). Hurricane Katrina in 2005 caused $\$ 108$ bn damage which amounts to $0.825 \%$ of GDP in the USA. Typhoon Haiyan in the Philippines in 20013 caused $\$ 2.8$ bn damage, equivalent to $1.05 \%$ of GDP.
} 
by the carbon tax in Golosov et al. (2014).

A drawback of this preliminary calculation is that the damage intensity and the abatement efficiency are not disentangled and, in fact, their calibration depends on the value of $\lambda$ and $\varepsilon$. In an attempt to provide a more rigorous estimation of $\theta$, we turn to the statistics on global carbon emissions and damages caused by major natural disasters. According to Reuters and the data by the World Bank, global $\mathrm{CO}_{2}$ emissions in 2013 amounted to 36 bn metric tons or equivalently to $(36 / 3.67=) 9.8092$ bn tons of carbon. With overall damages from severe natural disasters climbing over $\$ 90 \mathrm{bn}$, the damage intensity is calibrated as $\gamma_{e}=9.175(=90 / 9.8092)$ dollars per ton carbon. With the global world output in 2013 at $\$ 74.17172$ trillion, the polluting intensity is calibrated as $\phi=0.1322$ tons carbon per thousand dollars. The total factor productivity is set at $7 \%$ and $\sigma=1$. This calibration implies an abatement share of $0.9316 \%$ and a growth rate of $5.43 \%$. With more precise calibration of the climate damage intensity and polluting intensity we obtain a slightly higher estimate of $\theta$ and $g$. We find that an increase in the climate event arrival frequency by as little as $1 \%$ leads to more than doubling of the optimal abatement share, although the effect on the optimal growth rate is relatively small, only a 0.1 percentage points drop. Assuming a $5 \%$ lower intertemporal elasticity of substitution $(\varepsilon=1.05)$ leads to an increase in the abatement share by 0.63 percentage points and a decline in the optimal growth rate by 0.18 percentage points. On the other hand, assuming a slightly higher intertemporal elasticity of substitution $(\varepsilon=0.95)$ leads to a decline in $\theta$ to $0.238 \%$ and an increase in $g$ to $5.7208 \%$. Finally, a $5 \%$ increase in polluting intensity leads to a 0.66 percentage point increase in $\theta$ and a $0.05 \mathrm{pp}$ drop in $g$.

These numerical examples clearly illustrate the properties of the model and the importance of the climate change related parameters, although the numbers should be taken as suggestive rather than definitive. Specifically, varying crucial environmental parameters, such as the pollution intensity and the arrival rate of climate events, has a drastic impact on optimal policies. In a similar way, we confirm that the curvature of the utility function matters significantly for climate policy under uncertainty. Specifi- 
cally, the assumption of log-utility might limit the interpretation and applicability of the implied results in climate economics.

\section{Model Extensions}

\subsection{Adding Trend Fluctuations}

In this Section we extend the baseline model to a more general specification of the stochastic process for the capital stock. We shall assume that harmful emissions and the associated climate change cause not only jumps but also fluctuations around the trend. The latter are modeled by the Wiener process. Formally, the stochastic law of motion for the capital stock reads

$$
d K_{t}=\left[\left(1-\theta_{t}\right) Y_{t}-C_{t}\right] d t+b\left(E_{t}\right) d z_{t}-\gamma\left(E_{t}\right) d q_{t},
$$

where $d z$ is an increment of the standard Brownian motion, i.e., $z_{t}$ has mean zero and variance $t$, and $b_{t}=b_{z} E_{t}, b_{z} \in(0,1)$. The magnitude of the random fluctuations is linearly proportional to emissions with the proportionality parameter or "amplifier" $b_{z}$, assumed to be a small number. Given that $z_{t}$ has a normal distribution with mean zero, the deviations from the trend may be either positive or negative. The mechanisms by which GHG emissions may cause downward deviations from the trend are rather intuitive. They may be indirectly related to negative externalities caused by pollution to the economy's technology, total factor productivity, health status of the workforce, etc. Upward deviations from the trend, however, may also occur when (polluting) economic activity generates positive spillovers which cause the capital stock to increase. We do not model explicitly either of these deviations but take a shortcut through adopting the Brownian motion component in the stochastic representation of the capital stock path. The jump component remains the same as in the baseline setup, except that we set the exposure part $\bar{\gamma}=0$ for simplicity, so that $\gamma\left(E_{t}\right)=\gamma_{e} E_{t}, \gamma_{e}>0$. 
The HJB equation becomes

$$
\rho V(K)=\max _{C, \theta}\left\{u(C)+V^{\prime}(K)[(1-\theta) Y-C]+\frac{1}{2} V^{\prime \prime 2}(K)+\lambda[V(\widetilde{K})-V(K)]\right\}
$$

which now has an extra component - the second derivative of the value function - reflecting the presence of the Brownian motion. The optimality conditions with respect to the control and state variables are

$$
\begin{aligned}
C & : u^{\prime}(C)-V^{\prime}(K)=0 \\
\theta: & -V^{\prime}(K) Y-\frac{1}{2} V^{\prime \prime}(K) 2 b_{z}^{2}(\phi-\sigma \theta) Y^{2} \sigma+\lambda V^{\prime}(\widetilde{K}) \gamma_{e} \sigma Y=0 \\
K & : \rho V^{\prime}(K)=V^{\prime \prime}(K)\left[\left(1-\theta_{t}\right) Y-C\right]+V^{\prime}(K)(1-\theta) A+ \\
& +\frac{1}{2}\left[V^{\prime \prime \prime}(K)+V^{\prime \prime}(K) 2 b(\phi-\sigma \theta) b_{z} A\right]+\lambda\left[V^{\prime}(\widetilde{K})\left[1-\gamma_{e}(\phi-\sigma \theta) A\right]-V^{\prime}(K)\right] .
\end{aligned}
$$

We show in the Appendix that the optimality conditions yield

$$
\begin{gathered}
\frac{d C}{C}=\frac{1}{\varepsilon}\left\{A\left(1-\frac{\phi}{\sigma}\right)-\right. \\
\left.-\lambda-\lambda+\frac{1}{\sigma \gamma_{e}}-\frac{\varepsilon}{\widetilde{\psi}} b_{z}^{2} \frac{(\phi-\sigma \theta)}{\gamma_{e}}+\frac{\varepsilon(1+\varepsilon)}{2 \widetilde{\psi}^{2}} b_{z}^{2}(\phi-\sigma \theta)^{2}\right\} d t+ \\
+\frac{\varepsilon}{\widetilde{\psi}} b_{z}(\phi-\sigma \theta) d z+\varepsilon\left(\frac{\widetilde{C}}{C}-1\right) d q,
\end{gathered}
$$

where

$\widetilde{\psi}=\frac{\psi^{b}}{A}, \quad \psi^{b}=\frac{C}{K}=\frac{1}{\varepsilon}\left[\rho-(1-\varepsilon)(1-\theta) A+\frac{1}{2} \varepsilon(1-\varepsilon) b_{z}^{2}(\phi-\sigma \theta)^{2} A^{2}-\lambda \gamma_{e} A(\phi-\sigma \theta)\right]$.

We define the trend growth rate as

$$
g^{b} \equiv \frac{1}{\varepsilon}\left\{A\left(1-\frac{\phi}{\sigma}\right)-\rho-\lambda+\frac{1}{\sigma \gamma_{e}}-\frac{\varepsilon}{\widetilde{\psi}} b_{z}^{2} \frac{(\phi-\sigma \theta)}{\gamma_{e}}+\frac{\varepsilon(1+\varepsilon)}{2 \widetilde{\psi}^{2}} b_{z}^{2}(\phi-\sigma \theta)^{2}\right\}
$$

where the last two terms inside the parentheses depend on the abatement share $\theta$. The 
optimal abatement share solves

$$
\varepsilon b_{z}^{2}(\phi-\sigma \theta) \sigma A+\lambda \sigma \gamma_{e}\left[1-\gamma_{e}(\phi-\sigma \theta) A\right]^{-\varepsilon}=1,
$$

which is a non-linear equation in $\theta$ with two positive roots. Setting $b_{z}$ to zero, we are back to our baseline model with the unique solution for $\theta$ given by $\theta^{*}$ in Eq. (14) (with $\bar{\gamma}$ set to zero). Defining $x=\phi-\sigma \theta$, we can rewrite (26)

$$
1-\varepsilon b_{z}^{2} x \sigma A=\lambda \sigma \gamma_{e}\left(1-\gamma_{e} x A\right)^{-\varepsilon}
$$

To ensure the existence of a meaningful solution for a wide range of $\varepsilon$, we require that the term in the parentheses on the RHS is positive ${ }^{8}$ or $x<\frac{1}{\gamma_{e} A}$.

Proposition 4: When the evolution of the economy's capital stock is affected by climate change induced random jumps (via Poisson process) and trend fluctuations (via Wiener process),

(i) the optimal abatement expenditure represents a constant share of output;

(ii) the economy's trend growth rate is constant;

(iii) the abatement share is larger, while the growth may be either faster or slower, as compared to the baseline scenario (with only random jumps).

Proof: Follows from the discussion below.

Given that eq. (27) contains only constant terms, the implied abatement share, call it $\theta^{b}$, depends only on the parameters of the model and is therefore not time dependent. The solution to (27) is illustrated in Figure 2, where the left-hand side is shown by the downward-sloping straight line with the intercept at 1 and the right-hand side is shown by the hyperbola with the asymptote at $\frac{1}{A \gamma_{e}}$ and an intercept at $\lambda \sigma \gamma_{e}$. The two roots are given by the intersection of the straight line with the two parts of the hyperbola and are shown by $x_{1}$ and $x_{2}$. Note that, depending on the parameter values, the slope

\footnotetext{
${ }^{8}$ If the term happens to be negative and $\varepsilon \in(0,1)$, the RHS may become a complex number, an outcome which we do not wish to study further.
} 


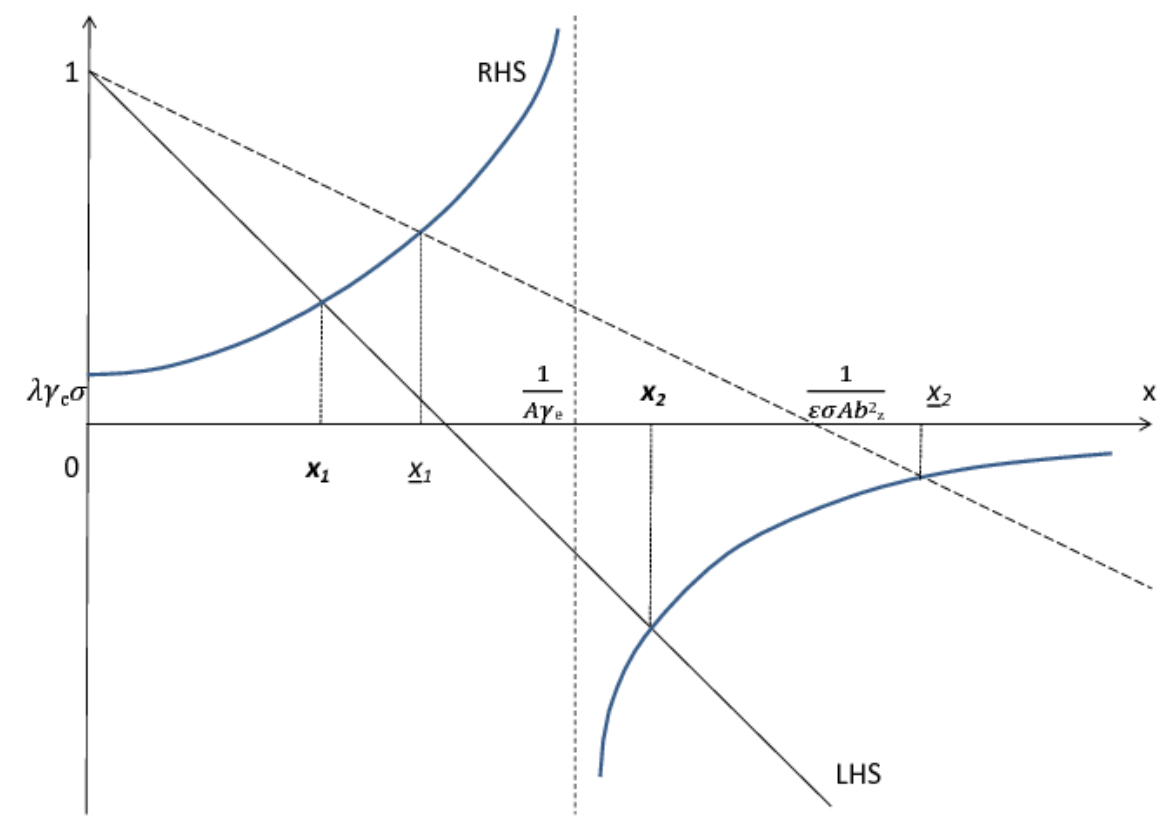

Figure 2: Solution to non-linear equation with two positive roots.

of the LHS may be either greater or smaller than unity, the latter case shown by the dashed line intersecting the $\mathrm{x}$-axes at $\frac{1}{\varepsilon \sigma A b_{z}^{2}}$. The roots are then as indicated by $\underline{x}_{1}$ and $\underline{x}_{2}$. Regardless of the slope value of LHS, one of the roots always lies in $\left(0, \frac{1}{A \gamma_{e}}\right)$, while the other root is always greater than $\frac{1}{A \gamma_{e}}$ and is therefore not considered further.

A constant $\theta^{b}$ implies a constant $\psi^{b}$ and thus the optimal consumption is a constant share of the capital stock (and of output as well). The optimal trend consumption growth $\left(g^{b}\right)$ is then also constant. Comparing the trend growth rates of our baseline model with the current solution we have:

$$
g^{b} \gtrless g \quad \Leftrightarrow \quad \theta^{b} \lessgtr \frac{\phi}{\sigma}-\frac{2 \psi^{b}}{A \gamma_{e} \sigma(1+\varepsilon)},
$$

where $\theta^{b}$ is the solution of $(27)$.

In the case of logarithmic utility (setting $\varepsilon=1$ ) the roots can be found explicitly by 
solving the quadratic equation

$$
\gamma_{e}\left(A b_{z}\right)^{2} \sigma x^{2}-\left(\gamma_{e}+b_{z}^{2} \sigma\right) A x+1-\lambda \sigma \gamma_{e}=0
$$

with

$x_{1}=\frac{\gamma_{e}+b_{z}^{2} \sigma-\sqrt{\left(\gamma_{e}-b_{z}^{2} \sigma\right)^{2}+4\left(\gamma_{e} b_{z} \sigma\right)^{2} \lambda}}{2 \gamma_{e} A b_{z}^{2} \sigma}, x_{2}=\frac{\gamma_{e}+b_{z}^{2} \sigma+\sqrt{\left(\gamma_{e}-b_{z}^{2} \sigma\right)^{2}+4\left(\gamma_{e} b_{z} \sigma\right)^{2} \lambda}}{2 \gamma_{e} A b_{z}^{2} \sigma}$.

Given our requirement $x<\frac{1}{\gamma_{e} A}$, the second root is eliminated, so that the corresponding solution for the abatement share is $\theta^{b}=\frac{\phi-x_{1}}{\sigma}$. It is thus possible to characterize the range of values of the fluctuations amplifier $b_{z}$ such that $g^{b}$ is greater or less than $g$. Using (29) and the definition of $\psi^{b}$, we obtain

$$
g^{b} \gtrless g \quad \Leftrightarrow \quad \gamma_{e}+b_{z}^{2} \sigma-\frac{2 A b_{z}^{2} \sigma \rho}{1+\lambda} \gtrless \sqrt{\left(\gamma_{e}-b_{z}^{2} \sigma\right)^{2}+4\left(\gamma_{e} b_{z} \sigma\right)^{2} \lambda} .
$$

In general, there are two cases to distinguish, depending on whether the LHS of the latter inequality is positive or negative, i.e., $\gamma_{e}(1+\lambda)+b_{z}^{2} \sigma(1+\lambda-2 A \rho) \gtrless 0$. In Case (1), the parameter constellation is such that the inequality is strictly negative. The LHS of Eq. (30) is then always smaller than the RHS. Thus the restriction on $b_{z}$ becomes

$$
b_{z}^{2}>\frac{\gamma_{e}(1+\lambda)}{\sigma[2 A \rho-(1+\lambda)]} \equiv \tau_{1}
$$

which defines the threshold $\tau_{1}$. If (the square of) the amplifier is above this threshold, $g^{b}$ is unambiguously lower than $g$. Intuitively, when polluting emissions have a relatively strong effect on the magnitude of fluctuations in the path of the capital stock, the economy's growth rate is lower than in a scenario without such fluctuations. Given that the amplifier is bounded from above by unity, the parameter space is restricted to $(1+\lambda)\left(1+\frac{\gamma_{e}}{\sigma}\right)<2 A \rho$. This condition also ensures that $\tau_{1}>0$. We note that this parameter constellation is realistically rather unlikely (although theoretically possible) since the LHS is a product of two numbers greater than unity, while the RHS is 2 times 
a product of relatively small numbers - the marginal productivity of capital and the rate of time preference.

We turn next to Case (2) such that the LHS of (30) is positive, i.e., $\gamma_{e}(1+\lambda)+$ $b_{z}^{2} \sigma(1+\lambda-2 A \rho)>0$. Raising both sides of (30) to the power of two and solving for $b_{z}$ yields another threshold value, $\tau_{2}$ :

$$
g^{b} \gtrless g \quad \Leftrightarrow \quad b_{z}^{2} \lessgtr \frac{\gamma_{e}(1+\lambda)\left[\left(1-\gamma_{e} \sigma \lambda\right)(1+\lambda)-A \rho\right]}{A \rho \sigma(1+\lambda-A \rho)} \equiv \tau_{2}
$$

It can be verified that $\tau_{2}<\tau_{1}$ if they are both positive. Two subcases are possible, depending on the parameter constellation: either $0<\tau_{2}<\tau_{1}<1$ or $\tau_{1}<0<\tau_{2}<1$.

Case (2a): $\quad g^{b}<g \Leftrightarrow \tau_{2}<b_{z}^{2}<\tau_{1}$, and $g^{b}>g \Leftrightarrow 0<b_{z}^{2}<\tau_{2}$, when

$$
\left(\frac{1+\lambda}{2}\right)\left(1+\frac{\gamma_{e}}{\sigma}\right)<A \rho<(1+\lambda)\left(1-\gamma_{e} \sigma \lambda\right) \text {. }
$$

Case (2b): $\quad g^{b}>g \Leftrightarrow 0<b_{z}^{2}<\tau_{2}$, and $g^{b}<g \Leftrightarrow \tau_{2}<b_{z}^{2}<1$, when

$$
\left\{\begin{array}{l}
A \rho<\min \left\{\frac{1+\lambda}{2},(1+\lambda)\left(1-\gamma_{e} \sigma \lambda\right)\right\} \\
A \rho \in\left(z_{1}, z_{2}\right), z_{1,2}=\frac{(1+\lambda)\left[\gamma_{e}+\sigma \pm \sqrt{\left(\gamma_{e}-\sigma\right)^{2}+4 \sigma^{2} \gamma_{e}^{2} \lambda}\right]}{2 \sigma} .
\end{array}\right.
$$

Intuitively, when climate change induced jumps and trend fluctuations are both present, the growth rate of the economy is smaller than under the baseline scenario if the fluctuations amplifier is relatively large - above $\tau_{1}$ in Case (1), between $\tau_{2}$ and $\tau_{1}$ in Case (2a), and between $\tau_{2}$ and unity in Case (2b). If the amplifier is relatively small, so that emissions do not cause large swings in the capital stock, the trend growth rate of the economy is enhanced.

Even though the optimal growth rate may be either greater or smaller than in the baseline, the optimal abatement share, $\theta^{b}$, is unambiguously greater than $\theta^{*}$. To see this, first note that $(27)$ is decreasing in $\theta$. Then insert $\theta^{*}$ in the equation and find that the LHS becomes smaller than the RHS. In order for the two to be equal again, $\theta$ must increase. Thus, $\theta^{b}>\theta^{*}$. The intuition is that now $\theta^{b}$ must absorb an additional source of uncertainty. Since the fluctuations are amplified by greenhouse gas emissions, there 
is a need to increase resources devoted to abatement.

Total differentiation of (27) allows to draw insights on the optimal response of the abatement share to changes in the climate parameters of the model

$$
\begin{aligned}
\frac{d \theta^{b}}{d b_{z}} & =\frac{2 b_{z} x}{\sigma\left[b_{z}^{2}+\lambda \gamma_{e}^{2}\left(1-\gamma_{e} x A\right)^{-\varepsilon-1}\right]}>0, \\
\frac{d \theta^{b}}{d \gamma_{e}} & =\frac{\lambda\left(1-\gamma_{e} x A\right)^{-\varepsilon-1}\left[1-\gamma_{e} x A(1-\varepsilon)\right]}{\varepsilon \sigma A\left[b_{z}^{2}+\lambda \gamma_{e}^{2}\left(1-\gamma_{e} x A\right)^{-\varepsilon-1}\right]}>0 .
\end{aligned}
$$

As expected, an increase in both damage-intensity parameters requires an unambiguous increase in the optimal abatement share.

\subsection{Stock Pollution}

In our baseline model we assumed that climate change induces natural disasters and the associated damages are larger, the larger is the flow of polluting emissions. In this extension we explore the implications of the assumption that damages depend on the accumulated stock of pollution, labeled $P_{t}$. We now model damages to the capital stock by the function $\gamma\left(P_{t}, K_{t}\right)=\gamma_{p} P_{t}+\bar{\gamma} K_{t}$, with $\gamma_{p}$ being the new damage intensity parameter which may, in general, be either greater or smaller than $\gamma_{e}$ of the baseline model, although it may be safe to assume that the marginal damage impact of the stock should be at least as large as that of the flow, implying $\gamma_{p} \geqslant \gamma_{e}$. The dynamics of the pollution stock are described by the differential equation

$$
d P_{t}=\left(E_{t}-\alpha P_{t}\right) d t
$$

The parameter $\alpha \in[0,1)$ represents the natural absorption rate of greenhouse gases and $E_{t}$ stands for the current emissions as before. The new stochastic law of motion for the capital stock is then given by

$$
d K_{t}=\left[(1-\theta) Y_{t}-C_{t}\right] d t-\gamma\left(P_{t}, K_{t}\right) d q_{t}
$$


and the capital stock after occurrence of an event is $\widetilde{K}=K-\gamma(P, K)$. We generalize the representative agent's utility function to include both consumption and pollution stock, reflecting the fact that individuals generally dislike environments with high levels of pollution. For simplicity, we shall assume a standard CRRA additively-separable function where consumption and pollution enter symmetrically, ${ }^{9} U(C, P)=\frac{C^{1-\varepsilon}-\chi P^{1-\varepsilon}}{1-\varepsilon}$.

The problem is to maximize the expected present discounted utility subject to (31), (32) and the two constraints of the baseline model, reproduced for convenience

$$
\begin{aligned}
& E_{t}=\phi Y_{t}\left(K_{t}\right)-\sigma I_{t}, \\
& I_{t}=\theta Y_{t}\left(K_{t}\right),
\end{aligned}
$$

where $\theta$ is the share of output devoted to abatement. The value function in the HamiltonJacobi-Bellman equation has now two arguments - the capital and the pollution stocks:

$$
\rho V(K, P)=\max _{C, \theta}\left\{U(C, P)+V_{k}[(1-\theta) Y-C]+V_{p}[(\phi-\theta \sigma) Y-\alpha P]+\lambda(\widetilde{V}-V)\right\}
$$

where we omitted the time subscripts and used the notation $V_{k} \equiv \partial V(K, P) / \partial K, V_{p} \equiv$ $\partial V(K, P) / \partial P$. The first-order conditions include:

$$
\begin{array}{ll}
C: \quad U_{c}-V_{k}=0, \\
\theta: \quad-V_{k} Y-V_{p} \sigma Y=0, \\
K: \quad \rho V_{k}=V_{k k}[(1-\theta) Y-C]+V_{k}(1-\theta) A+V_{p}(\phi-\sigma \theta) A+V_{p k}[(\phi-\sigma \theta) Y-\alpha P]+ \\
\quad+\lambda\left(\widetilde{V}_{k}(1-\bar{\gamma})-V_{k}\right), \\
P: \quad \rho V_{p}=U_{p}+V_{p p}[(\phi-\sigma \theta) Y-\alpha P]+V_{k p}[(1-\theta) Y-C]-\lambda \gamma_{p} \widetilde{V}_{k}-\alpha V_{p}+ \\
\quad+\lambda\left(\widetilde{V}_{p}-V_{p}\right) .
\end{array}
$$

Computing the differentials of $V_{k}$ and $V_{p}$ allows us to obtain a system of two equations

\footnotetext{
${ }^{9}$ Although the symmetrical structure is not common in the literature, it allows us to obtain an explicit analytical solution. A slight disadvantage of this type of utility function is that the disutility of pollution is decreasing at a lower incremental rate in pollution stock.
} 
in two unknowns - the differential of consumption and the ratio of marginal utilities of consumption before and after the jump. We define the post-to-pre-jump consumption ratio as $\widetilde{\omega}$ and may write:

$$
\begin{gathered}
\frac{\tilde{U}_{c}}{U_{c}}=\widetilde{\omega}^{-\varepsilon}=\frac{A\left(1-\frac{\phi}{\sigma}\right)+\alpha+\sigma \frac{U_{p}}{U_{c}}}{\lambda\left(\sigma \gamma_{p}+\bar{\gamma}\right)}, \\
g^{p} \equiv \frac{d C}{C}=\frac{1}{\varepsilon}\left\{A\left(1-\frac{\phi}{\sigma}\right)-\rho+\lambda\left[\widetilde{\omega}^{-\varepsilon}(1-\bar{\gamma})-1\right]\right\},
\end{gathered}
$$

where $g^{p}$ defines the trend consumption growth rate. The appropriate guess of the value function allows us to prove the following:

Proposition 5: Compared to the baseline setting where the climate damage is a function of emissions flow, in a setting where the climate damage depends on the whole history of pollution,

(i) the consumption rate is a constant fraction of the capital stock,

(ii) the consumption jump, due to an adverse climate event, is smaller and

(iii) the trend growth rate is smaller.

Proof: (i) follows directly from the first-order conditions and the optimal value function $V(K, P)=\frac{X_{1} K^{1-\varepsilon}}{1-\varepsilon}-\frac{X_{2} P^{1-\varepsilon}}{1-\varepsilon}$. The two constants $X_{1}$ and $X_{2}$ are functions of the parameters of the model and are determined by solving the following system of two non-linear equations, where $x \equiv\left(\frac{X_{2}}{X_{1}}\right)^{1 / \varepsilon}$ is the relative weight of pollution in the value function:

$$
\left\{\begin{aligned}
& x\left(\chi \sigma^{\frac{1-\varepsilon}{\varepsilon}} X_{2}^{-1}-\rho-(1-\varepsilon) \alpha \sigma^{\frac{1-\varepsilon}{\varepsilon}}\right)=\varepsilon x X_{2}^{-\frac{1}{\varepsilon}}+(1-\varepsilon) A\left(1-\frac{\phi}{\sigma}\right)-(\rho+\lambda)+ \\
&+\lambda\left(1-\bar{\gamma}-\gamma_{p} \sigma^{1 / \varepsilon} x\right)^{1-\varepsilon} \\
& \frac{A\left(1-\frac{\phi}{\sigma}\right)+\alpha-\frac{\chi}{X_{2}}}{\lambda\left(\sigma \gamma_{p}+\bar{\gamma}\right)}=\left(1-\bar{\gamma}-\gamma_{p} x \sigma^{1 / \varepsilon}\right)^{-\varepsilon} .
\end{aligned}\right.
$$

It follows that the consumption-capital ratio is constant and given by $X_{1}^{-1 / \varepsilon}$.

(ii) The optimal ratio of post- to pre- jump consumption rate is obtained by substi- 
tuting the optimal controls for $U_{p} / U_{c}$ in Eq. (35)

$$
\bar{C}=\left[\frac{\lambda\left(\sigma \gamma_{p}+\bar{\gamma}\right)}{A\left(1-\frac{\phi}{\sigma}\right)+\alpha-\frac{\chi}{X_{2}}}\right]^{1 / \varepsilon} .
$$

It is clear that the term in the denominator is between zero and unity, while in the numerator $\gamma_{p} \geqslant \gamma_{e}$, and therefore $\widetilde{\omega}>\omega$, implying that the consumption jump is smaller than under the baseline scenario of Section 2 .

(iii) Comparing the expression for the trend growth rate in Eq. (36) with Eq. (18), we find that $g^{p}<g$ since $\widetilde{\omega}^{-\varepsilon}<\omega^{-\varepsilon}$.

It follows from Proposition 5 that the optimal consumption under "pollution-stock scenario" exhibits a time profile with less pronounced jumps and less vigorous growth. This is because in this case (as opposed to the baseline) an arrival of a climate shock causes a larger damage to the capital stock, since the damage depends on the accumulated pollution and not just on the current level. Therefore, a relatively slow accumulation of pollution, associated with a slower growth, becomes optimal.

In a special case of logarithmic utility, the system (37) can be solved explicitly for the constants $X_{1}$ and $X_{2}$. For notational convenience, define the economy's implicit interest rate as $r \equiv A\left(1-\frac{\phi}{\sigma}\right)+\alpha$, then

$$
X_{2}=\frac{x(\chi-1)}{\rho(x-1)}
$$

and $x$ is the solution to the quadratic equation

$x^{2}[\rho \chi-(\chi-1) r] \sigma \gamma_{p}+x\left\{(\chi-1)\left[r(1-\bar{\gamma})-\lambda\left(\bar{\gamma}+\sigma \gamma_{p}\right)\right]-\rho \chi\left(1-\bar{\gamma}+\sigma \gamma_{p}\right)\right\}+\rho \chi(1-\bar{\gamma})=0$.

Focusing on the unique root and setting $\bar{\gamma}=0, \chi=1 / 2$ for simplicity, we have

$$
x=\frac{r+\rho+\sigma \gamma_{p}(\rho-\lambda)}{2 \sigma \gamma_{p}(r+\rho)},
$$




$$
X_{1}=\frac{\sigma \gamma_{p}(r+\rho)}{\rho\left[(r+\rho)\left(2 \sigma \gamma_{p}-1\right)-\sigma \gamma_{p}(\rho-\lambda)\right]}, \quad X_{2}=\frac{r+\rho+\sigma \gamma_{p}(\rho-\lambda)}{2 \rho\left[(r+\rho)\left(2 \sigma \gamma_{p}-1\right)-\sigma \gamma_{p}(\rho-\lambda)\right]}
$$

Inserting the solution for $X_{2}$ into (38) and the result in (36), we obtain the optimal trend growth rate of consumption

$$
g^{p}=r-\alpha-(\rho+\lambda)+\frac{r+\rho-\sigma \gamma_{p}(\rho+\lambda)}{\sigma \gamma_{p}\left[r+\rho+\sigma \gamma_{p}(\rho-\lambda)\right]}
$$

The responses of the optimal growth rate to changes in the key climate-change parameters, such as $\lambda, \gamma_{p}$ and $\sigma$ are provided below:

$$
\begin{aligned}
\frac{d g^{p}}{d \alpha} & =\frac{2 \rho}{\left[r+\rho+\sigma \gamma_{p}(\rho-\lambda)\right]^{2}}>0, \\
\frac{d g^{p}}{d \phi} & =-\left\{1+\frac{2 \rho}{\left[r+\rho+\sigma \gamma_{p}(\rho-\lambda)\right]^{2}}\right\} \frac{A}{\sigma}<0, \\
\frac{\partial g^{p}}{\partial \lambda} & =-1-\frac{2 \rho \sigma \gamma_{p}}{\left[r+\rho+\sigma \gamma_{p}(\rho-\lambda)\right]^{2}}<0, \\
\frac{\partial g^{p}}{\partial \gamma_{p}} & =-\frac{(r+\rho)\left[r+\rho+\sigma \gamma_{p}(\rho-\lambda)\right]+\sigma \gamma_{p}(\rho-\lambda)\left[r+\rho-\sigma \gamma_{p}(\rho+\lambda)\right]}{\sigma \gamma_{p}^{2}\left[r+\rho+\sigma \gamma_{p}(\rho-\lambda)\right]^{2}}<0, \\
\frac{d g^{p}}{d \sigma} & =\frac{\left(A \phi \gamma_{p}-\sigma^{2}\right)\left[r+\rho+2 \sigma \gamma_{p}(\rho-\lambda)\right](r+\rho)+\left(\sigma \gamma_{p}\right)^{2}(\rho-\lambda)\left[\rho\left(A \phi \gamma_{p}+\sigma^{2}\right)-\lambda\left(A \phi \gamma_{p}-\sigma^{2}\right)\right]}{\sigma^{2} \gamma_{p}\left[r+\rho+\sigma \gamma_{p}(\rho-\lambda)\right]^{2}} \gtrless 0 .
\end{aligned}
$$

Similarly to the baseline model, a higher pollution intensity of production, a larger disaster arrival rate and a higher damage intensity slow down the growth, while the abatement efficiency has an ambiguous effect. The ambiguity is due to the two counteracting forces. The first is the positive effect of $\sigma$ on the implicit interest rate $r$, which translates into the effect on growth via $\frac{\partial g^{p}}{\partial r} \frac{\partial r}{\partial \sigma}=\left\{1+\frac{2 \rho}{\left[r+\rho+\sigma \gamma_{p}(\rho-\lambda)\right]^{2}}\right\} \frac{A \phi}{\sigma^{2}}>0$. The second is the negative effect of $\sigma$ on the consumption jump - a better abatement efficiency reduces the magnitude of the consumption drop when an event occurs. This effect is given by the term $\frac{\partial g^{p}}{\partial \sigma}=-\frac{\sigma^{2} \gamma_{p}(\rho-\lambda)\left[2(r+\rho)-\sigma \gamma_{p}(\rho+\lambda)\right]+(r+\rho)^{2}}{\gamma_{p}\left[r+\rho+\sigma \gamma_{p}(\rho-\lambda)\right]^{2}}<0$. If the magnitude of the positive effect of $\sigma$ on the interest rate is larger than the inverse of the damage intensity, i.e., $\frac{A \phi}{\sigma^{2}}>\frac{1}{\gamma_{p}}$, and the arrival rate does not exceed the rate of time preference, i.e., $\rho \geqslant \lambda$, then the overall effect $d g^{p} / d \sigma$ is unambiguously positive. 
We turn next to the optimal share of output devoted to abatement activities. The optimality condition for the choice of $\theta$ and the optimal value function imply that the trend growth rates of both capital and pollution stocks are the same. The growth rate of the pollution stock is easily obtained from Eq. (31), so that we have

$$
g^{p}=\left(\frac{\phi}{\sigma}-\theta\right) \frac{A}{x}-\alpha
$$

and after rearranging and using the solution for $x$

$$
\theta=\frac{\phi}{\sigma}-\frac{r+\rho+\sigma \gamma_{p}(\rho-\lambda)}{2 \sigma \gamma_{p}(r+\rho) A}\left(g^{p}+\alpha\right)
$$

Note that the abatement share depends directly on the economy's trend growth rate. The effects of the key climate parameters on the abatement share have the same sign as those of the baseline model and their magnitudes are as follows:

$$
\begin{aligned}
& \frac{d \theta}{d \lambda}=\frac{2+\sigma \gamma_{p}(r-\rho-\lambda)}{2 A \sigma \gamma_{p}(r+\rho)}>0, \\
& \frac{d \theta}{d \gamma_{p}}=\frac{1+r+\rho+\sigma \gamma_{p}(\rho-\lambda)}{2 A \sigma^{2} \gamma_{p}^{3}}>0 .
\end{aligned}
$$

The abatement efficiency $\sigma$ affects $\theta$ through three channels. First directly through the first term in Eq. (39) - the negative effect. Second, through the relative weight of pollution in the value function $x$ - the negative effect; and finally through the growth rate - either positive or negative effect. Therefore, the overall effect of $\sigma$ on the abatement share is ambiguous, as in our baseline model.

\section{Conclusions}

Using a theoretical growth model we derive closed-form solutions for the impact of uncertain climate change on economic development and optimum climate policies. We show that the model's efficiency and preference parameters have the expected effects both on 
economic dynamics as well as on optimum climate mitigation. Importantly, we find that both the arrival rate of natural disasters and the size of the damages unambiguously decrease the growth rate while having strong positive effects on optimal abatement efforts. From our general setup including climate uncertainty it thus emerges that capital accumulation and optimum abatement react significantly to disaster size and frequency. To achieve a social optimum, mitigation policies have to be increased significantly when climate impact rises.

There are two main implications of our results for the current climate policy debate. First, unlike in the deterministic analysis, we are able to provide results for the realistic case of disaster frequencies, which are conceptually different from averages of expected events. The analysis shows that the impact on optimum mitigation policies is very large, because in social optimum the economy aims at avoiding climate-induced growth losses. Therefore, basing mitigation policies on the deterministic framework of the cost-benefit analysis clearly underestimates optimum mitigation expenditures. Second, given the fact that less developed countries are more vulnerable to climate change, it becomes clear that the climate impact for those countries is especially severe. Instead of converging to higher income standards they are hit by consumption reductions which may turn out to be percentage-wise larger than those of the developed world. Moreover, they need to use a larger share of their income for abatement instead of accumulating capital and catching up with the wealthy economies. Because climate change is a global environmental problem and the world community is committed to development goals for all the countries on the globe, mitigation policies should be undertaken or financed by the advanced economies. 


\section{References}

[1] Baryshnikov, Y., A. Mayo, and D.R. Taylor (2001): Pricing of CAT Bonds, unpublished manuscript.

[2] Batabyal, A.A. and H. Beladi (2001): Aspects of the Theory of Financial Risk Management for Natural Disasters, Applied Mathematics Letters, 14, 875-880.

[3] Bovenberg, A.L. and S. Smulders (1995): Environmental Quality and Pollutionaugmenting Technological Change in a Two-sector Endogenous Growth Model, Journal of Public Economics, 57, 369-391.

[4] Bommier, A., B. Lanz, and S. Zuber (2013): Models-as-Usual for Unusual Risks? On the Value of Catastrophic Climate Change, Working Paper, ETH Zurich

[5] Bretschger, L. and S. Valente (2011): Climate Change and Uneven Development, Scandinavian Journal of Economics, 113/4, 825-845.

[6] Bretschger, L. and N. Suphaphiphat (2013): Effective Climate Policies in a Dynamic North-South Model, European Economic Review, in Press.

[7] Brock, W. A. and M. S. Taylor (2005): Economic Growth and the Environment: A Review of Theory and Empirics, in: Handbook of Economic Growth, S. Durlauf and P. Aghion (Eds.), Vol. 1, Chapter 28: 1749-1821, Elsevier.

[8] Cai, Y., K. L. Judd, and T. S. Lontzek (2013): The social cost of stochastic and irreversible climate change, NBER Working Paper 18704.

[9] Clarke, H. R. and W. J. Reed (1994): Consumption/Pollution Tradeoffs in an Environment Vulnerable to Pollution-Related Catastrophic Collapse, Journal of Economic Dynamics and Control, 18: 991-1010.

[10] Economist (2013): Cyclones and climate change: The new normal? Nov 16th, 2013

[11] Golosov, M., J. Hassler, P. Krusell and A. Tsyvinski (2013): Optimal Taxes on Fossil Fuels in General Equilibrium, Econometrica, forthcoming. 
[12] Grimaud, A., G. Lafforgue and B. Magné (2007): Economic Growth and Climate Change in a Decentralized Economy: A Theoretical and Empirical Approach, LERNA Working Paper 07.04.225, University of Toulouse

[13] Ikefuji, M. and R. Horii, (2012): Natural disasters in a two-sector model of endogenous growth, Journal of Public Economics 96, 784-796

[14] Ikefuji, M., R.J.A. Laeven, J.R. Magnus, and C. Muris (2010): Expected Utility and Catastrophic Risk in a Stochastic Economy-Climate Model, CentER Discussion Paper No. 2010-122, Tilburg University.

[15] IPCC (2007): Fourth Assessment Report, Contribution of Working Group II, M.L. Parry, O.F. Canziani, J.P. Palutikof, P.J. van der Linden and C.E. Hanson (eds), Cambridge University Press, Cambridge, UK.

[16] Lemoine, D. and C. Traeger (2013): Watch your step: Optimal policy in a tipping climate, American Economic Journal: Economic Policy, forthcoming.

[17] Lontzek, T. and D. Narita (2011): Risk-Averse Mitigation Decisions in an Unpredictable Climate System, Scandinavian Journal of Economics 113/4, 937-958.

[18] Michel, P. and G. Rotillon (1995): Disutility of Pollution and Endogenous Growth, Environmental and Resource Economics, 6, 279-300.

[19] Nordhaus, W.D. (2008): A Question of Balance: Weighing the Options on Global Warming Policies. Yale University Press, New Haven.

[20] Nordhaus, W.D. and J. Boyer (2000): Warming the World. MIT Press, Cambridge.

[21] Pindyck, R.S. (2012): Uncertain outcomes and climate change policy, Journal of Environmental Economics and Management 63, 289-303

[22] Rezai, A., D.K. Foley, and L. Taylor (2012): Global Warming and Economic Externalities, Economic Theory, 49, 329-351.

[23] Soretz, S. (2007): Efficient Dynamic Pollution Taxation in an Uncertain Environment, Environmental and Resource Economics 36, 57-84. 
[24] Sennewald, K. and K. Wälde (2006): "Itô's Lemma" and the Bellman Equation for Poisson Process: An Applied View, Journal of Economics, 89, 1-36.

[25] Steger, T.M. (2005): "Stochastic Growth under Wiener and Poisson Uncertainty," Economics Letter, 86, 311-316.

[26] Toche, P. (2005): "A Tractable Model of Precautionary Saving in Continous Time," Economics Letters, vol. 87, no. 2: 267-272.

[27] Tsur, Y. and A. Zemel (1996): Accounting for global warming risks: Resource management under event uncertainty, Journal of Economic Dynamics and Control, $20,289-1305$.

[28] Tsur, Y. and A. Zemel (1998): Pollution control in an uncertain environment, Journal of Economic Dynamics and Control, 22: 967 - 975.

[29] Van der Ploeg, F. and C. Withagen (2010): Growth, Renewables, and the Optimal Carbon Tax, OxCarre Research Paper 55, University of Oxford.

[30] Wälde, K. (1999): Optimal Saving under Poisson Uncertainty, Journal of Economic Theory 87, 194-217.

[31] Withagen, C. (1995): Pollution, Abatement and Balanced Growth, Environmental and Resource Economics, 5, 1-8.

[32] de Zeeuw, A. and A. Zemel (2012): Regime Shifts and Uncertainty in Pollution Control, Journal of Economic Dynamics and Control, 36: 939-950. 